\title{
Longitudinal monitoring of whole body counter NaI(Tl) detector efficiency
}

R. J. Shypailo

USDA/ARS Children's Nutrition Research Center, Department of Pediatrics, Baylor College of Medicine, Houston, TX 77030

\section{Correspondence to:}

Roman J. Shypailo

Children's Nutrition Research Center

Baylor College of Medicine

1100 Bates Street

Houston, TX 77030, USA

Fax: 713.798.7094

Tel: 713.798.7127

Email: shypailo@bcm.edu

Submitted to: Applied Radiation and Isotopes 


\title{
Longitudinal monitoring of whole body counter $\mathrm{NaI}(\mathrm{Tl})$ detector efficiency
}

\author{
R. J. Shypailo
}

USDA/ARS Children's Nutrition Research Center, Department of Pediatrics, Baylor College of Medicine, Houston, TX 77030

\begin{abstract}
Assessing accuracy of radiation counting systems over time is critical. We examined long-term WBC performance in detail. Efficiency factors for 54 detectors were updated annually over several years. Newer efficiency values were compared with baseline and with annual values. Overall system efficiency has declined (-1.9\% over 3 yrs) and appears to be doing so at an increasing rate. Having more specific performance data on individual components can make the process of system maintenance and repair more straightforward and efficient.
\end{abstract}

\section{Key words:}

Body composition; Calibration; Gamma rays; Monte Carlo method; Whole-body counting; Efficiency; Reproducibility of Results

\section{Summary}

Continued assessment and evaluation of radiation counting systems in order to maintain a consistent degree of accuracy over time is important, yet there is very little in 
the literature focusing on longitudinal empirical monitoring of scintillation detector performance. We wished to examine the long-term performance of the whole body counter (WBC) at the Children's Nutrition Research Center (CNRC) in Houston in more detail, above that provided by normal quality control $(\mathrm{QC})$ procedures. Each detector $(\mathrm{n}=54)$ was initially assigned an efficiency correction factor, derived from comparisons against an ideal reference detector defined by Monte Carlo modelling. These correction factors were updated annually by measuring a 60Co NIST-traceable calibration point source of known activity. This annual assessment has been done for the past several years. We compared these annual findings with the initial efficiency calibration values, and have tracked changes for each detector. Newer efficiency values were compared with baseline and with the previous year's values. Detectors were evaluated individually and as a group. Overall, the system appears fairly stable, with a relative standard deviation of less than one percent; yet, certain individual detectors exhibit a significant trend of declining efficiency over time. The overall WBC efficiency has declined in recent years (-1.9\% over 3 yrs), and appears to be doing so at an increasing rate. Annual adjustment of detector correction factors allows the system to produce consistent results even though individual components may be changing. More detailed monitoring beyond normal or cursory quality control measures may be beneficial to maintain the integrity of system accuracy. Having more specific performance data on individual components can make the process of system maintenance and repair more straightforward and efficient. 


\section{Introduction}

Scintillation detectors such as those composed of NaI(Tl) or HPGe are commonly used for assessment of ionizing radiation for a variety of applications. Whole body counters incorporating $\mathrm{NaI}(\mathrm{Tl})$ detectors are used in body composition studies for assessment of total body potassium (Shypailo and Ellis 2011, Shukla et al 1973). Other types of radiation counters are commonly used in nuclear medicine, often in conjunction with positron emission tomography (PET) or more commonly with single-photon emission computed tomography (SPECT). These techniques are used to measure cerebral blood flow and tissue metabolism, plasma volume, various kinetic studies, to list just a few examples (Blake et al 2013, Lodge et al 2015). In the field of radiation safety, in vivo monitoring of potential internal contamination of radionuclides within the human body is carried out using gamma detectors (Breustedt et al 2011). Aside from monitoring internal radiation from the human body, radiation counting systems are being more commonly used for the detection of explosives or contraband materials, and in fuel analysis (Gordon and Peters 1990, Vourvopoulos and Womble 2001, Burr and Hamada 2009).

A common thread tying together these disparate applications is the need for system calibration and for quality control. Radiation detection systems must be initially calibrated to assess counting efficiency, converting accumulated counts to measured activity; and systems must be monitored regularly to ensure continued accuracy and dependability.

Perhaps the most common approach to system calibration involves the use of Monte Carlo particle transport modeling coupled with phantom standards containing known amounts of radioisotopes. One can easily find many articles in the literature outlining such calibration efforts leading to improved precision and accuracy (Shypailo 
and Ellis 2011, Bhati et al 2012, Krstic and Nikezic 2012). Normally, the work presented involves initial system calibration, or recalibration on the heels of system reconfiguration; yet, there is very little in the literature focusing on longitudinal assessment of detector performance. This sort of continued assessment and evaluation may be important in ensuring that a system maintains the same degree of accuracy throughout its working lifespan. The relevance of this is underlined in a study by Ishikawa (2000) during the course of a 24-yr longitudinal assessment of $40 \mathrm{~K}$ in Japanese males. Variations in data due to changes in $\mathrm{NaI}(\mathrm{Tl})$ detector sensitivity necessitated the periodic replacement of detectors in order to maintain system stability. Details concerning the specifics of detector evaluation are not provided, but the work serves as an example of research that can be affected by long term instrument degradation.

Certainly, quality control efforts are normally incorporated into any measurement instrument, including radiation counting systems (Breustedt et al 2011, Matheoud et al 2012). While quality control monitoring often focuses on a system in its entirety, we were interested in reviewing the performance of our whole body counter (WBC) in more detail. The WBC housed in the Children's Nutrition Research Center (CNRC) at Baylor College of Medicine in Houston uses $54 \mathrm{NaI}(\mathrm{Tl})$ detectors for the assessment of total body potassium (TBK) (Shypailo and Ellis 2011), an index of body cell mass (Moore 1963). High precision and accuracy of the system is critical to its success in measuring a variety of subjects for many research protocols. The system was put in place and initial studies began in 2004. Work on the WBC continued, with various improvements made, and by 2008 the system could be considered as finalized and stable. 
Data on the performance of the individual detectors has been accumulated for several years, and annual assessment of each detector was instituted after the first few years in an attempt to monitor and adjust for detector aging and degradation. Thus, calibration corrections could be made on a regular basis in order to maintain a consistent counting efficiency. Our goal was to review and present these data, offering an insight into the overall performance of the CNRC WBC. While perhaps directly applicable only to this type of counter, the approach and findings may be of benefit to users of most any radiation measurement system. In addition, these types of findings are an invaluable aid in the decision making process related to the replacement of critical system components.

\section{Methods}

The CNRC WBC has been described previously (Shypailo and Ellis 2011). In summary, the counter uses $54 \mathrm{NaI}(\mathrm{Tl})$ detectors, arrayed in two symmetrical banks of 27 detectors each, positioned above and below a bed. The detectors, $10 \mathrm{~cm} \mathrm{x} 10 \mathrm{~cm}$ square $\mathrm{x}$ $7.6 \mathrm{~cm}$ thick, were manufactured by Scionix Holland B.V. (Bunnik, Netherlands). The detectors are housed in a shielded cylindrical tunnel. In addition, each detector array is inset into a steel lattice, which results in moderate collimation and further background reduction. The steel lattice surrounding a detector can be seen in Figure 1. The overall WBC efficiency calibration was done using MCNP modeling software (MCNP-5; Los Alamos National Laboratory) (Briesmeister 1986). In addition, each detector was first modeled and calibrated for its individual efficiency, similar to the approach presented by O’Hehir et al (2006). 
In order to calculate this individual detector efficiency factor, the Scionix detector was first modeled as precisely as possible using MCNP (Figure 1). The model was finetuned so that empirical results matched model predictions for a single reference detector. Data were collected from a 60Co NIST-traceable calibration point source of known activity (Eckert \& Ziegler Isotope Products, Valencia, CA). The source was positioned at a fixed distance from the detector face, and counts were accumulated for $200 \mathrm{sec}$. Once the MCNP model was finalized, spectra were acquired for each of the 54 detectors in turn, with the 60Co calibration source positioned as in the MCNP model. The source to detector distance differed for upper bank and lower bank detectors - upper bank distance was 33 $\mathrm{cm}$ and lower bank distance was $28.7 \mathrm{~cm}$ - thus the model was adjusted accordingly. Three 200 second runs were acquired per detector and averaged to produce a customized efficiency correction factor. The correction factors represent the ratio of measured to expected counts for every detector. These factors are applied to every WBC potassium measurement in order to individually adjust detector counts since the MCNP model expects all detectors to be as efficient as the modeled reference detector.

Energy calibration was done on each detector individually using the 60Co source. Detector voltage and gain were adjusted in order to position the 60Co peaks at a consistent channel setting, thus all detectors display peaks from radioisotopes of interest at a common location on the energy axis. Quality control routines are run regularly to ensure that detectors maintain this fixed energy calibration.

Routine measurements of 40K QC standards allow us to track and maintain system stability. These QC standards are in the form of a distributed array of 27 plastic tubes, each $10 \mathrm{~cm}$ in length, and each containing approximately $65 \mathrm{~g}$ of $\mathrm{KCl}$ (for a total of about $1.8 \mathrm{~kg}$ 
$\mathrm{KCl})$. The tubes are affixed to plastic panels and placed onto the scanning bed at a constant position for each measurement. Measurements are done twice a week.

To track longitudinal detector performance, the same efficiency calibration procedure was carried out annually on each detector - the same 60Co source was measured three times for 200 seconds at the specified modeled distance. In other words, the exact same process as the initial detector efficiency calibration testing was carried out on all 54 detectors. This assessment has been done annually for the past several years.

We compared these annual findings with the initial efficiency calibration values, and have tracked changes for each detector. Newer efficiency values were compared with baseline and with the previous year's values. Detectors were evaluated individually and as a group. Summary data from the $54 \mathrm{WBC}$ detectors are separated into the upper and lower bank groups. The initial calibration and subsequent annual assessment of the detectors in each group were done using two different Monte Carlo models. The detector representations for each detector bank model were identical, but the source to detector distances were not. Thus, the WBC can in effect be considered as having two separate components - upper and lower detector groups - and the stability data were evaluated separately when presenting summary results.

Group differences were tested for statistical significance using the nonparametric paired Wilcoxon signed rank test, as the correction factors were not always normally distributed. Overall mean system efficiency as well as changes from previous years were assessed using linear regression in order to assess system stability. All statistical analyses were performed using XLSTAT Version 2016.04 (Addinsoft, New York, NY). For all 
statistical comparisons, a P-value (probability measuring the strength of evidence in support of a null hypothesis) of $<0.05$ was considered significant.

\section{Results}

Routine monitoring results for the WBC as a whole suggest a stable system, with an overall relative standard deviation of less than one percent $(<1.0 \%)$ for the measurement of 40K QC standards. A sample of QC results for each bank of detectors, representing approximately one year of monitoring, is shown in Figure 2. Linear regression lines for the detector groups do not show a significant trend $(\mathrm{P}=0.302$ and 0.131 for the upper and lower banks, respectively) over a one-year time period.

Annual efficiency testing results are shown in Figures 3 and 4 as bar graphs, divided into upper and lower bank detector groups. The set of bars for each individual detector represent sequential annual efficiency values for the years 2013 through 2016 . Detectors identified by a star $(*)$ exhibit a significant decreasing linear trend over time. Three such detectors are in the upper bank, and nine such detectors are in the lower bank. The remaining 42 detectors, while showing some variability, do not change significantly over time.

The overall annual mean efficiency values for the WBC for the past several years are listed in Table 1. The routine of annual detector efficiency testing was started in 2013, and the overall value at that time point did not differ from the previous assessment done five years earlier (2008) - both values were 0.970 . 
Since 2013, the overall WBC efficiency has declined each year at an increasing rate. Linear regression for the annual percent change over time shows a significant downward trend with a coefficient of determination $\left(\mathrm{R}^{2}\right)$ value of $0.955(\mathrm{P}=0.023)$.

A more detailed look at yearly changes in the system is presented in Table 2. Annual efficiency factors - representing the mean values for each bank of 27 detectors are shown along with the associated P-values comparing the detector groups against previous years and against the baseline year (i.e. 2008). Upper bank detectors as a whole appear to be more stable, differing from baseline only in the last year (2016), although a difference was detected when compared to the previous year in 2015. The lower bank detector group exhibited statistically significant differences one year earlier, starting with 2014 for the previous year comparison and 2015 for the baseline comparison.

\section{Discussion}

Modeling individual detectors and instituting regular efficiency evaluations provides an opportunity for tracking the performance of system components more thoroughly than overall system QC testing. The fairly large number of $\mathrm{NaI}(\mathrm{Tl})$ detectors in our WBC may necessitate this type of detailed testing, as many other WBC systems make use of fewer detectors (Krstic and Nikezic 2012, Paiva et al 2016, Bhati et al 2012). It may be easier to isolate and identify problems within these simpler systems, but it seems reasonable to assume that more detailed performance evaluations would benefit them as well.

Other investigators have presented work evaluating system performance. Lodge $e t$ al (2015) discuss calibration and standardization, and track stability for 100 days. Their 
intent, however, is still focused on establishing accurate calibration procedures rather than long term performance assessment.

Bhati et al (2012) describe an MCNP-based calibration using multiple phantom sizes to accommodate the variation in human body size and shape in their population, similar to the work done by Shypailo et al for the CNRC WBC (Shypailo and Ellis 2011). Their focus is on detection efficiency of the scanning system. Quality control is not addressed.

Certain factors that affect detector performance have been investigated by others. For example, Ianakiev et al (2009) point out the effects that temperature variations would have on detector light output, as well as stating that observable drift due mainly to PMT gain can occur over several weeks. Both of these effects are addressed in the CNRC system. Temperature is not a factor since the CNRC WBC is located indoors in a climate controlled area; while shorter term (i.e. weeks) drift is corrected as needed based on our biweekly QC standard runs. One can see several points in Figure 2 that lie outside of our acceptable standard deviation range (the dotted lines). These occurrences serve to call attention to potential detector drift or other maintenance issues and are normally promptly addressed in order to keep the system properly calibrated. In other words, these are shortterm incidents that are dealt with and corrected. Thus, our main concern is changes occurring over a much longer period of time.

It is generally accepted that scintillation detectors will experience degradation while aging due to numerous mechanisms, such as thermooxidative processes, mechanical stresses, and molecular diffusion, in addition to the stresses caused by radiation (Zhou et al 2011). Some investigators have explored the negative effects of these processes and 
addressed issues such as detector lifetimes, although the work has been invariably theoretical, involving simulations or predictive equations (Zhou et al 2011, Zolochevsky et al 2008). It is difficult to find empirical studies that have focused on detector aging.

Although it is well known that $\mathrm{NaI}(\mathrm{Tl})$ is highly hygroscopic in addition to being fragile (Melcher 2000, IAEA 2003), we can only speculate that increasing crystal hydration is responsible for our observed deteriorating detector performance. It is interesting to note that the lower bank detector group was seemingly more susceptible to degradation, as three times as many lower bank detectors vis-à-vis upper bank detectors (9 vs 3) showed significantly reduced counting efficiency over our measurement time period. It does not seem likely that humidity levels would differ significantly between the spaces occupied by lower and upper bank detectors. One real difference, however, is the physical orientation of the detectors within each bank. Upper bank detectors rest on the edges surrounding the front detector face, while lower bank detectors rest on the housing surrounding the photomultiplier. The different mechanical stresses induced by these arrangements may be involved in effecting detector performance, but at this point the data to confirm this is unavailable.

Much attention is devoted to detector calibration and description and to developing detector response functions, all with the goal of improving performance to identify and to quantify radioisotopes (Burr and Hamada 2009, Sood and Gardner 2004, Rogers 1982). In our WBC, this has been addressed using the combined approach of Monte Carlo modeling along with meticulous empirical testing. Routine QC for energy calibration then follows as an important task to ensure that variations in detector results are not due to gain shifts and spectrum drifts. 
As noted by Ceccatelli et al (2010), counting efficiency is dependent on the radioisotope of interest, the geometrical and source efficiencies, and lastly on the intrinsic detector characteristics - the topic of most interest to this discussion. It is this last factor that looks to be changeable with time and should be monitored if one intends to maintain a dependable counting efficiency.

The benefits of the approach presented here are twofold: 1) regular adjustment of calibration factors to ensure consistent accuracy, and 2) evaluation of individual detector performance to identify potential sources of error. The annual adjustment of detector correction factors that are summarized in Table 2 allows the system to produce consistent results even though individual components may be changing. Had we not made these annual corrections, 2016 results for TBK would appear to be almost $2 \%$ lower than they would have been in 2013. These would not have been real changes. These inaccuracies would be due strictly to decreasing detector efficiency. Additionally, if these downward performance trends continue, accuracy errors would become even more pronounced without any calibration factor intervention.

Since TBK is used as an index of body cell mass, and is predictive of skeletal muscle mass, even calibration errors of $2 \%$ could have a significant effect on study outcomes and conclusions if not corrected. WBC precision and sensitivity are crucial when studying various diseases and monitoring growth and health. For example, while measuring TBK, investigators have observed a loss of muscle mass and lean tissue with normal aging of $0.5 \%$ to $1 \%$ annually - a value that can vary with weight, and gender and accelerate with age (Kehayias et al 1997, Kyle et al 2001). Over the three year time period represented in our study, older individuals may exhibit a $1.5 \%$ to $3 \%$ loss of muscle mass, 
which is roughly comparable to the potential measurement errors due to uncorrected efficiency factors in our system. Thus a loss of sensitivity of this magnitude would have a significant effect on verifying real losses in muscle mass. In contrast to studies showing decreasing TBK with age, a more recent longitudinal study by Andreoli et al (2010) representing a 21-yr period showed relatively stable levels of TBK amidst increasing body weight. As with the previous studies, changes in detector efficiency over such a time period could lead to faulty conclusions of TBK changes, such as decreases where there are none.

It is also important to note that a simple summary measure such as a relative standard deviation $(0.5 \%$ overall for this system for a period covering one year and $<1.0 \%$ for a multi-year period) may be deceiving. Repeatability of a measurement with less than a one percent deviation is certainly quite good. Yet individual components within that system showed signs of degradation that could only be detected by more detailed analysis and tracking.

Longitudinal monitoring of individual detectors allows us to identify units most in need of attention and maintenance. Figure 4 identifies several detectors showing significant trends of decreasing counting efficiency. Candidates for repair or replacement would most likely come from this group. Detector 32, for example, shows both significant efficiency loss and a somewhat low efficiency factor (0.88 in 2016) compared to other detectors. It would be prudent to focus on a detector such as this if component replacement is being considered. Similarly, observing the patterns of change may provide clues to detector performance. Detector 44 did not show a significant change in efficiency statistically, yet 
the efficiency drop in the last year looks to be worrisome. It may be prudent to monitor this detector more closely until the next annual assessment.

One can see in Table 1 that the overall efficiency of the CNRC WBC may be declining at an accelerated rate - values for percent change from year to year are increasing. If this trend continues, it may be necessary to evaluate the system more frequently, perhaps by changing the annual to a biannual efficiency testing. This would require us to adjust the calibration factors more frequently in order to maintain system accuracy. In addition, future work may focus on identifying the cause of the efficiency losses, i.e. the detectors themselves, vis-à-vis the supporting electronics or related systems.

\section{Conclusion}

Our approach to system calibration, using Monte Carlo modeling, allowed us to derive and apply individual detector calibration factors. Annual, or more frequent, reassessment allows us to adjust and correct these calibration factors to maintain accuracy. Other investigators may have designed and calibrated their systems differently, yet regular assessment of the performance of individual components can still be carried out, as long as that assessment is standardized and reproducible. Judging by the data presented here, one may expect to see changes and perhaps degradation of system performance over time. More detailed monitoring beyond normal or cursory quality control measures may be beneficial to maintain the integrity of system accuracy. Having more specific performance data on individual components can make the process of system maintenance and repair more straightforward and efficient. 


\section{Acknowledgments}

We are grateful to M Laurent for performing the standard QC measurements and annual detector evaluations for the CNRC WBC. This work is a publication of the USDA/ARS Children's Nutrition Research Center, Department of Paediatrics, Baylor College of Medicine, and Texas Children's Hospital, Houston, TX. Funding has been provided from the USDA/ARS under Cooperative Agreement No. 58-6250-6-001. The contents of this publication do not necessarily reflect the views or policies of the USDA, nor does mention of trade names, commercial products, or organizations imply endorsement by the US Government.

\section{References}

Andreoli, A., Volpe, S., Ratcliffe, S., Daniele, N., Imparato, A., Gabriel, L., Parente, G., Possemato, S., Lorenzo, A., 2010. Longitudinal Study of Total Body Potassium in Healthy Men. J Am Coll Nutr 29, 352-356.

Bhati, S., Patni, H.K., Ghare, V.P., Singh, I.S. and Nadar, M.Y., 2012. Monte Carlo calculations for efficiency calibration of a whole-body monitor using BOMAB phantoms of different sizes. Radiat Prot Dosimetry 148(4), 414-419 doi:10.1093/rpd/ncr203.

Blake, P., Johnson, B., VanMeter, J.W., 2003. Positron Emission Tomography (PET) and Single Photon Emission Computed Tomography (SPECT): Clinical Applications. J Neuroophthalmol 23, 34-41. 
Breustedt, B., Mohr, U., Biegard, N., Cordes, G., 2011. Quality management system and accreditation of the in vivo monitoring laboratory at Karslruhe Institute of Technology. Radiat Prot Dosimetry 144(1-4), 95-97 doi:10.1093/rpd/ncq363.

Briesmeister, J.F., 1986. MCNP - A general Monte Carlo code for neutron and photon transport. Los Alamos Report LA-7396-M, Rev. 2.

Burr, T. and Hamada, M., 2009. Radio-Isotope Identification Algorithms for NaI $\gamma$ Spectra. Algorithms 2, 339-360 doi:10.3390/a2010339.

Ceccatelli, A. ,Campanella, F., Ciofetta, G., Marracino, F.M., Cannat, V., 2010. A new experimental procedure for determination of photoelectric efficiency of a $\mathrm{NaI}(\mathrm{Tl})$ detector used for nuclear medicine liquid waste monitoring with traceability to a reference standard radionuclide calibrator. Appl Radiat Isot 68, 275-279 doi:10.1016/j.apradiso.2009.10.041.

Gordon, C.M. and Peters, C.W., 1990. A fast-neutron probe for tomography and bulk analysis. Intern J Appl Radiat Isot 41, 1111-1116.

International Atomic Energy Agency. Quality control atlas for scintillation camera systems. IAEA STI/PUB/1141, Vienna 2003.

Ianakiev, K.D., Alexandrov, B.S., Littlewood, P.B., Browne, M.C., 2009. Temperature behavior of $\mathrm{NaI}(\mathrm{Tl})$ scintillation detectors. Nucl Instrum Meth A607, 432-438 doi:10.1016/j.nima.2009.02.019.

Ishikawa, T., 2000. Long-term changes in body potassium concentration and dose from 40K for a Japanese adult male group. Health Phys 78, 420-33.

Kehayias, J.J., Fiatarone, M.A., Zhuang, H., Roubenoff, R., 1997. Total body potassium and body fat: relevance to aging. Am J Clin Nutr 66, 904-10. 
Krstic, D. and Nikezic, D., 2012. Efficiency of whole-body counter for various body size calculated by MCNP5 software. Radiat Prot Dosimetry 152(1-3), 179-183 doi:10.1093/rpd/ncs219.

Kyle, U., Genton, L., Hans, D., Karsegard, L., Slosman, D., Pichard, C., 2001. Age-related differences in fat-free mass, skeletal muscle, body cell mass and fat mass between 18 and 94 years. Eur J Clin Nutr 55, 663-672.

Lodge, M.A., Holt, D.P., Kinahan, P.E., Wong, D.F., Wahl, R.L., 2015. Performance assessment of a $\mathrm{NaI}(\mathrm{Tl})$ gamma counter for PET applications with methods for improved quantitative accuracy and greater standardization. EJNMMI Phys. 2, 11 doi:10.1186/s40658-015-0114-3 .

Matheoud, R., Goertzen, A.L., Vigna, L., Ducharme, J., Sacchetti, G., Brambilla, M., 2012. Five-year experience of quality control for a 3D LSO-based whole-body PET scanner: Results and considerations. Physica Medica 28, 210-220 doi:10.1016/j.ejmp.2011.07.002.

Melcher, C.L., 2000. Scintillation crystals for PET. J Nucl Med 41, 1051-5.

Moore, F.D., Boyden, C.M., 1963. Body cell mass and limits of hydration of the fat-free body: their relation to estimated skeletal weight. Ann N Y Acad Sci 110, 62-71.

O’Hehir, S., Green, S., Beddoe, A.H., 2006. A new method of body habitus correction for total body potassium measurements. Phys Med Biol 51, 4253-4265 doi:10.1088/0031$9155 / 51 / 17 / 009$. 
Paiva, F.G., Oliveira, A.H., Mendes, B.M., Pinto, J.R., Filho, N.D., Dantas, B.M., Dantas, A.L., Silva, T A., Lacerda, M.A., Fonseca, T.C., 2016. Improvement of the WBC calibration of the Internal Dosimetry Laboratory of the CDTN/CNEN using the physical phantom BOMAB and MCNPX code. Appl Radiat Isot 117, 123-127 doi:10.1016/j.apradiso.2015.12.059.

Rogers, D., 1982. More realistic Monte Carlo calculations of photon detector response functions. Nucl Instrum Meth Phys Res 199, 531-548.

Shukla, K.K., Ellis, K.J., Dombrowski, C.S., Cohn, S.H., 1973. Physiological variation of total-body potassium in man. Am J Physiol 224, 271-4.

Shypailo, R.J., Ellis, K.J., 2011. Whole body counter calibration using Monte Carlo modeling with an array of phantom sizes based on national anthropometric reference data. Phys Med Biol 56, 2979-97 doi:10.1088/0031-9155/56/10/006.

Sood, A., Gardner, R.P., 2004. A new Monte Carlo assisted approach to detector response functions. Nucl Instrum Meth Phys Res Section B: Beam Interactions with Materials and Atoms 213, 100-104.

Vourvopoulos, G. and Womble, P.C., 2001. Pulsed fast/thermal neutron analysis: a technique for explosives detection. Talanta. 54(3), 459-468 doi:10.1016/S00399140(00)00544-0.

Zhou, T., He, H., Sheng, X., 2011. The long-term stability of plastic scintillator for electromagnetic particle detectors. Proceedings of the 32nd International Cosmic Ray Conference (ICRC2011) Beijing, China 4, 346-349 doi:10.7529/ICRC2011/V04/0267. 
Zolochevsky, A.A., Goncharova, G.V., Borodenko, Y.A., Kozmin, Y.S., 2008. .Simulation of the scintillation detector degradation process. Journal of National Technical University - Kharkov Polytechnic Institute 9, 79-89. 


\section{Figure Captions}

Fig. 1 MCNP detector model. *Source size and position not to scale. Source to detector distance: Upper Bank $=33 \mathrm{~cm}$; Lower Bank $=28.7 \mathrm{~cm}$.

Fig. 2 Routine QC results using 40K standards. Results depict upper bank (A) and lower bank (B) detectors. Dotted lines indicate $+/-2 \mathrm{x}$ standard deviation of the mean of each group. Solid lines represent linear regression trends.

Fig. 3 Annual detector test results showing efficiency factors (measured/expected counts) for Upper Bank detectors. Starred (*) detectors exhibit a significant trend of declining efficiency over time. The bars represent the years 2013 to 2016 in sequence.

Fig. 4 Annual detector test results showing efficiency factors (measured/expected counts) for Lower Bank detectors. Starred (*) detectors exhibit a significant trend of declining efficiency over time. The bars represent the years 2013 to 2016 in sequence. 
Table 1. Mean annual efficiency factors for WBC system

\begin{tabular}{ccc}
\hline Year & Efficiency Factor & \%Change $^{\mathrm{a}}$ \\
\hline 2008 & 0.970 & - \\
2013 & 0.970 & $0.00 \%$ \\
2014 & 0.966 & $-0.40 \%$ \\
2015 & 0.959 & $-0.76 \%$ \\
2016 & 0.951 & $-0.88 \%$ \\
\hline
\end{tabular}

${ }^{\mathrm{a}}$ Values indicate the percentage change from the previous year.

Table 2. Year to year efficiency factor comparison for two detector banks

\begin{tabular}{|c|c|c|c|c|c|}
\hline Year: & 2008 & 2013 & 2014 & 2015 & 2016 \\
\hline \multicolumn{6}{|l|}{ UPPER BANK } \\
\hline Efficiency Factor: & 0.961 & 0.963 & 0.961 & 0.954 & 0.950 \\
\hline Annual Comparisor & -value: & 0.435 & 0.155 & $0.036^{\mathrm{a}}$ & 0.070 \\
\hline Baseline Comparis & P-value: & 0.435 & 0.639 & 0.234 & $0.004^{\mathrm{a}}$ \\
\hline \multicolumn{6}{|l|}{ LOWER BANK } \\
\hline Efficiency Factor: & 0.979 & 0.977 & 0.972 & 0.964 & 0.954 \\
\hline \multicolumn{2}{|c|}{ Annual Comparison P-value: } & 0.639 & $0.023^{\mathrm{a}}$ & $0.003^{\mathrm{a}}$ & $0.0002^{\mathrm{a}}$ \\
\hline \multicolumn{2}{|c|}{ Baseline Comparison P-value: } & 0.639 & 0.160 & $0.006^{\mathrm{a}}$ & $0.001^{\mathrm{a}}$ \\
\hline
\end{tabular}

${ }^{a}$ Values significantly different from the previous year (Annual) or from the initial year (Baseline). 
${ }^{60}$ Co Point Source*

Steel Shielding

\section{$\mathrm{Nal}(\mathrm{TI})$ Crystal}

Quartz Light Pipe

Stainless Steel Housing 
1.1

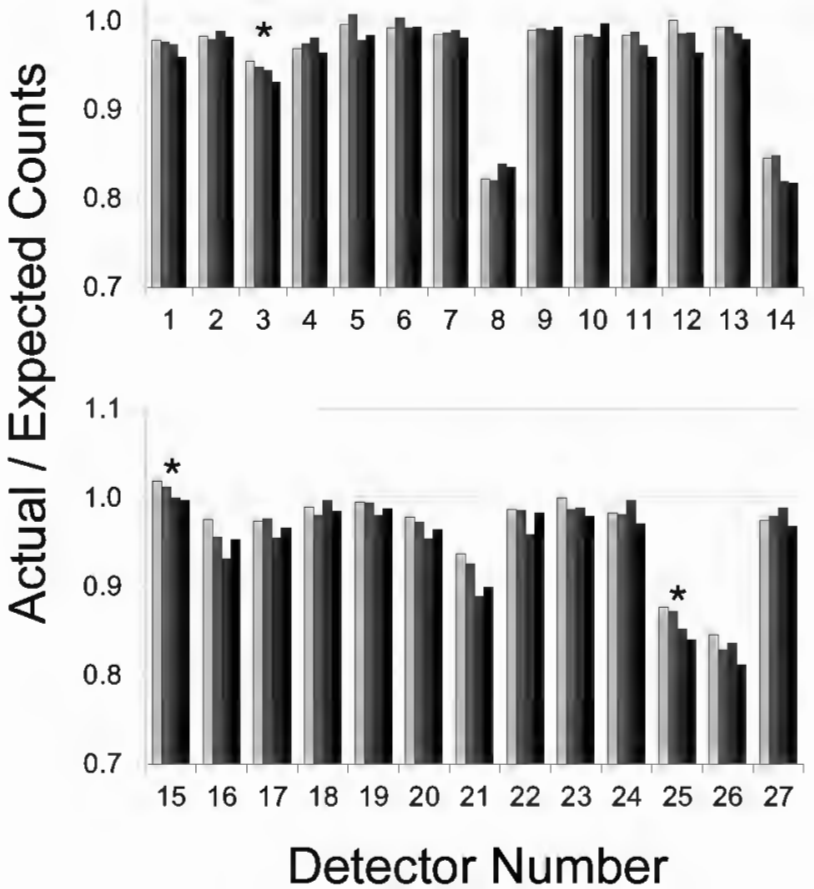


1.1

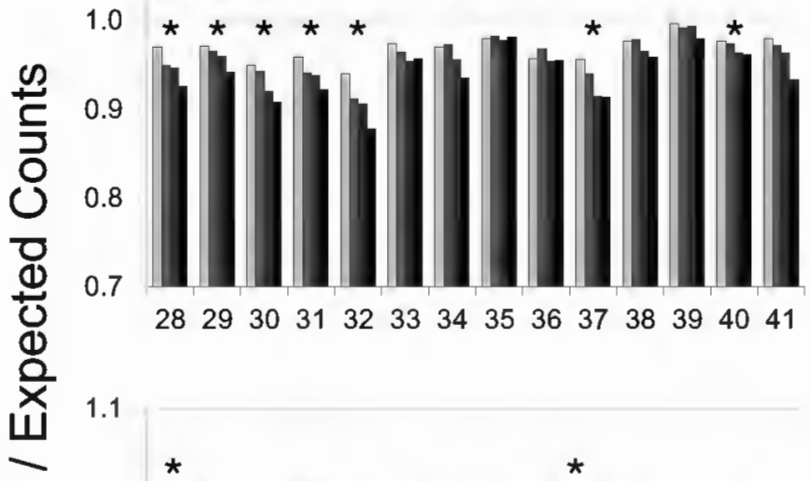

$\frac{\bar{\sigma}}{\frac{7}{0}}$

0.9

0.8

0.7

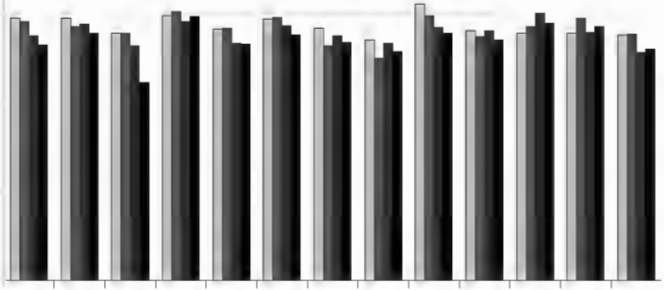

$\begin{array}{lllllllllllll}42 & 43 & 44 & 45 & 46 & 47 & 48 & 49 & 50 & 51 & 52 & 53 & 54\end{array}$ 